\title{
Distinct Roles of ROCK1 and ROCK2 on the Cerebral Ischemia Injury and Subsequently Neurodegenerative Changes
}

\author{
Weizhuo Lu $^{\text {a }}$ Jiyue Wen ${ }^{b}$ Zhiwu Chen ${ }^{b}$ \\ a School of Nursing, Hefei Technology College, Hefei, China; ${ }^{b}$ Department of Pharmacology, School of Basic Medical \\ Sciences, Anhui Medical University, Hefei, China
}

\section{Keywords}

ROCK1 $\cdot$ ROCK2 $\cdot$ Cerebral ischemia injury ·

Neurodegenerative changes

\begin{abstract}
Cerebral ischemic injury is one of the main causes of adult disability and death. Although significant progress has been made, cerebral ischemia continues to be a major risk to public health worldwide. The Rho kinase (ROCK) signaling pathway has been reported to be significantly involved in many mechanisms of cerebral injury. Although ROCK is ubiquitously expressed in all tissues, ROCK2 subtype expression in brain and the spinal cord is more abundant and improves with age. This makes it a promising target for new therapeutic approaches. In this article, we review the current knowledge on the involvement of ROCK in cerebral ischemia injury and neurodegenerative changes after cerebral injury. After a detailed description of the mechanism of ROCK involvement in axonal regeneration and synaptic function, different roles of ROCK1 and ROCK2 in neurons under physiological and pathological conditions are compared and discussed. In addition, different functions of genetic and pharmacological inhibitions of ROCK1 and ROCK2 on cerebral injury are discussed.

(c) 2019 S. Karger AG, Basel
\end{abstract}

\section{Introduction}

Rho-associated protein kinase (ROCK), downstream effector of Rho GTPases, is a kind of serine-threonine protein kinase. Two mammalian ROCK homologs, ROCK1 and ROCK2, have been identified, which share $92 \%$ identity of amino acid sequence in their kinase domain and have a similar structure [1]. In its native state, ROCK is self-suppressed by the $\mathrm{pH}$ domain in the carboxyl-terminal region. However, when RhoA and (or) RhoC are activated to bind to the RBD domain or the auto-inhibitory region is cleaved and removed by caspase-3 or Granzyme$B$, ROCK is activated [2].

ROCK affects numerous cellular processes such as cell contraction and motility in brain, many of them in endothelial cells, vascular smooth muscle, neurons, glia etc. These processes are related to the pathophysiology of cerebral ischemia injury, which makes ROCK a unique multifaceted objective of ischemic research and of various potentially synergistic mechanisms against ischemic injury [3]. Furthermore, ROCK, a ubiquitously expressed messenger, has been regarded as a therapeutic target in various other models of neurological diseases with sig-

J.W. and Z.C. contributed equally to this work.

\section{KARGER}

(๑) 2019 S. Karger AG, Basel

E-Mail karger@karger.com

www.karger.com/pha
Dr. Jiyue Wen and Dr. Zhiwu Chen

Department of Pharmacology

School of Basic Medical Sciences

Anhui Medical University, Hefei (China)

E-Mail wenjiyue139@aliyun.com and chpharmzw@163.com 
nificant success, such as traumatic brain injury [4], subarachnoid hemorrhage [5], spinal cord injury [6], neurodegenerative changes after cerebral injury [7], as well as a number of neurodegenerative diseases [8-12].

The restorative capacity of the central nervous system is strictly limited, and the inability of injured axon to reestablish intact projections leads to lasting functional lesion after acute cerebral stroke or chronic neurodegenerative diseases. The regeneration of injured neurons is inhibited by multiple factors, such as axonal degeneration, neuronal apoptosis, loss of regenerative substrate, reduced intrinsic growth state, and inhibitory environment [13]. Consequently, there is a pressing need to explore the mechanism of degeneration of neurons after injury and identify potential therapeutic targets for ischemic stroke for pharmaceutical intervention.

As a well-described signaling cascade transmission, RhoA/ROCK pathway is an inhibitory cue to the growth cone by modulation of actin dynamics [14]. Within this pathway, ROCK has a vital role on inhibitory signals for axon growth and in the regulation of cell survival [15]. Although ROCK is ubiquitously expressed in all tissues, ROCK1 is mainly expressed in non-neuronal tissue and ROCK2 is expressed in brain and the spinal cord abundantly and improves with age [16]. Thus, we summarize our current knowledge on ROCKs, especially the divergent contribution of ROCK1 and ROCK2 to the cerebral ischemia injury and neurodegenerative changes after cerebral injury.

\section{ROCK and Axonal Regeneration}

ROCK inhibition has been reported to alter the dendritic spine morphology in hippocampal neurons [17]. The activation of Rho/ROCK pathway, mediated by the binding of trimeric NogoR/p75/Lingo 1, semaphorin-3A $[18,19]$, ephrin-A [20] or semaphorin-4D [21] to their receptors, not only contributes to the developmental modulation of axonal guidance, but also plays a key role in a regenerative setting in the central nervous system, where developmental modulation of axonal guidance has been shown to be upregulated following injury [22], and finally leads to the retraction of neurites and growth cone collapse [23]. In addition to inhibition of regeneration, ROCK activation is also deleterious for cell viability [24,25].

As mentioned before, ROCK2 is expressed more prominently in neurons. It distributes abundantly in dendrites and neuron perikarya of the hippocampus, the frontal lobe, and the cerebellum, which are essential for understanding its contribution to essential brain functions [26]. Knockdown of ROCK2 expression in adult fe- male rats can be specifically attributed to the remarkable biological effects after optic nerve crush, such as attenuation of axonal degeneration, inhibition of apoptosis, and promotion of neurite outgrowth. Moreover, downregulation of ROCK2 decreased caspase-3 activity and calpain, increased autophagic flux, and attenuated acute axonal degeneration [27]. This emphasizes that ROCK2 has the potential to be a central therapeutic target for traumatic and degenerative nervous system disorders [28].

\section{ROCK and Intrinsic Membrane Properties and}

Synaptic Inputs

Myosin light chain (MLC) is one of the main substrates of ROCK, which is both directly and (or) indirectly phosphorylated. The other actions of MLC kinase and MLC phosphatase determine the level of MLC phosphorylation (MLCp), which are modulated by ROCK [29]. MLCp subsequently participates in the stimulation of actin-myosin interactions at presynaptic terminals [14].

ROCK regulates the electrical activity of neuronal circuits and neurotransmitter release by cytoskeletal-dependent modulation of the presynaptic vesicle pools, which is supported by the observation that the size of the pool of fast releasing vesicles is controlled by MLC kinase at the calyx of Held synapse [30]. This was further confirmed by the findings that ROCK modulates the motoneuron physiology via regulating synaptic inputs and/or intrinsic membrane properties, and regulates the presynaptic actin-myosin contractile apparatus to maintain a determined bulk of the readily releasable pool of synaptic vesicles in the baseline condition [26].

In addition, actin filaments, which are thin polymers of protein actin, form an intricate cytoskeletal network maintaining a close association with vesicles and active zones. Actin, in turn, prevents synapsin and capture vesicles from fusing to the plasma membrane by interacting with them [31]. ROCK, localized to the cytoplasm, is near the active zone, especially close to actomyosin filament bundles in presynapses, and modulates the interaction of myosin and actin through regulation of MLCp level [32].

Despite sharing high sequence similarity and downstream substrates between ROCK1 and ROCK2, ROCK2 is expressed at higher levels in brain, and the expression of ROCK2 in mouse brain gradually increases during the postnatal development [33]. In addition, ROCK2 in bovine brain is more profuse in neurons of hippocampus, cerebral cortex, and cerebellum [34]. Furthermore, genetic studies suggest that ROCK1 and ROCK2 have distinct functions, which are demonstrated using embryonic fibroblasts from $\mathrm{ROCK}^{-/-}$and $\mathrm{ROCK} 2^{-/-}$mouse in regulating the actin 
cytoskeleton. Research revealed that ROCK1 destabilized actin via phosphorylation of MLC, whereas ROCK2 stabilized actin by cofilin phosphorylation [35].

Thus, differential patterns of tissue distribution of ROCK1 and ROCK2 in brain suggest different cerebral functions. Hence, further studies are needed to assess the contributions of ROCK1 and ROCK2 to the synaptic function.

\section{ROCK and Hippocampal Synapses and \\ Learning-Memory Abilities}

The progressive impairment of learning and memory is believed to result from hippocampal synaptic dysfunction [36]. Synaptic dysfunction occurs from an early stage of neurodegenerative pathology, and there is accumulating evidence that synaptic dysfunction precedes neuronal loss. Thus, the signal transduction of synaptic dysfunction may be a vital indicator of the beginning stage of neurodegenerative pathology [37]. ROCK participates in spatial learning and memory and fear memory consolidation, which are dependent on its modulation of synaptic plasticity processes [38]. Furthermore, ROCK at the preand post-synaptic hippocampal synapse is important for long-lasting potentiation [39].

Cofilin, one of the downstream effectors of ROCK, is an important factor for regulating the synaptic plasticity, especially maintaining the actin cytoskeleton in dendritic spines [40]. Actin-binding proteins, cofilins play a central role in improving actin filament dynamics and recombination through severing and depolymerizing actin filaments at their pointed ends [41]. Decrease of severing activity can help maintain and promote motility of growth cone and extension of neurite [42]. ROCK controls cytoskeletal dynamics, which in part is dependent on the activation of LIM kinases (LIMK), and results in phosphorylation and inactivation of the cofilin [43]. Thus, ROCK inhibition is considered a promising target site for the prevention of synaptic degeneration [14], and in achieving some outstanding results, for instance, ROCK inhibitor, Fasudil has been reported to successfully ameliorate cognitive deficits in rats following cerebral ischemia [44] and in aged rats [38]. Furthermore, Fasudil can ameliorate the synaptic structure and function in streptozotocin-treated rat model of sporadic Alzheimer's disease through LIMK2 dephosphorylation and subsequent cofilin dephosphorylation (activation) [45].

In addition, several studies have reported that ROCK2 expression, but not ROCK1, is upregulated significantly in human nervous tissue from patients with neurodegenerative disorders [7]. Suppression of ROCK2 expression showed a promising foreground for the treatment of Alzheimer's disease [46]. Moreover, ROCK2 knockdown protects dopaminergic neurons in the substantia nigra pars compacta against degeneration induced by hydroxydopamine and increases the motor behavior of mouse model of Parkinson's disease [47]. All of these distinct functions of ROCK2 in the pathogenesis of neurodegenerative diseases may help determine whether selective ROCK inhibitors could be clinically useful. Therefore, it is desirable to develop inhibitors specifically affecting ROCK2 when targeting neurodegenerative diseases. However, chronic treatment with Fasudil, ROCK1 and ROCK2 inhibitors has been shown to induce anxiety-like behaviors [48]. This finding is necessary and will be of great benefit for the continued development of ROCK isoform-selective and pan-ROCK inhibitors to treat human diseases.

\section{ROCK and Chronic Cerebral Ischemia}

Ischemic stroke is the main cause of physical disability and mortality worldwide. Besides, the chronic cerebral ischemia, blood flow perfusion inadequacy over a long period of time can induce progressive or persistent cognitive dysfunction in patients [49]. ROCK activity generally increases not only in the endothelium, vascular smooth muscle cells, but also in the neurons [50] after ischemia stroke. As mentioned before, ROCK modulates neural cell proliferation, migration, axon guidance, and regeneration [26], and closely relates to the pathogenesis of a wide variety of nervous system diseases and disorders [51]. Furthermore, ROCK participates in the modulation of smooth muscle cell proliferation via increasing endothelial permeability, up-regulation of MLCp, and decreasing the expression of endothelial nitric oxide synthase [52-54], all of these ROCK actions after ischemia stroke could result in more serious cerebral blood supply disorders.

Not surprisingly, previous studies have reported that the expression and activation of ROCK2, but not ROCK1, improved gradually in brain tissues with ischemia time using rat model of cerebral chronic ischemia, which is in accordance with the cognitive dysfunction, and can be inhibited by Fasudil pre-treatment [55]. Furthermore, myosin-binding subunit is the first confirmed substrate of ROCK and is considered to be a marker of ROCK activation [56]. The expression of myosin-binding subunit has been found to elevate significantly in the brain tissues of chronic cerebral ischemia rats, peaked at 6 weeks, whose change was similar to the ROCK2 expression [55]. These findings further confirmed that the upregulation of ROCK2 expression could lead to cognitive dysfunction 
after chronic cerebral ischemia, and selective ROCK2 inhibitors may be a promising therapeutic use in the treatment of chronic cerebral ischemia.

\section{Inhibition of ROCK Expression}

Although ROCK1 and ROCK2 share protein substrates, such as LIMKs, MLC and MLC phosphatase, the distinct functions of ROCK isoforms are revealed by accumulated research $[57,58]$, using genetic approaches of ROCK1 or ROCK2 deletion [59]. For example, the differential effects of modulating the reorganization of actin cytoskeleton has been revealed in the working of embryonic fibroblasts of ROCK1 knockout $\left(\mathrm{ROCK}^{-/-}\right)$or ROCK2 knockout $\left(\mathrm{ROCK}^{-/-}\right)$mouse after doxorubicin exposure; ROCK1 destabilizes actin cytoskeleton through MLCp, by contrast, ROCK2 stabilizes actin cytoskeleton via cofilin phosphorylation [35]. Although the complications of homozygous ROCK1 and ROCK2 knockout mice are limited, reports from the genetic exploration of cerebral ROCK function indicated that ROCK heterozygosity $\left(\mathrm{ROCK}^{+/-}\right.$or $\mathrm{ROCK}^{+/-}$) could be used to define the ROCK isoformspecific functions of dendritic structural plasticity, injury by cerebral ischemia, loss of endothelial ROCK2 leading to enhanced endothelial function, higher cerebral blood flow, and greater neuroprotection $[16,60]$. Increased basal and apical dendritic length and dendritic intersections were discovered in $\mathrm{ROCK}^{+/-}$mice but not in $\mathrm{ROCK}^{+/-}$ones. Although the densities of dendritic spine were similar between 2 genotypes, length of apical spine was reduced in $\mathrm{ROCK}^{+/-}$mice but elevated in ROCK $2^{+/-}$ones. All of these observations implicated that ROCK $1^{+/-}$or ROCK $2^{+/-}$ mice can be used as a novel experimental animal for study and a detailed distinct function of ROCKs determined.

\section{ROCK Inhibitors}

ROCK inhibitors, hydroxyfasudil or Y-27632 has been demonstrated to be beneficial for stroke prevention, acute neuroprotection, and chronic stroke recovery. Inhibition of ROCK improved the neurologic deficit scores by $37 \%$, and reduced the cerebral infarct size by $33 \%$ [61]. Another nonselective ROCK inhibitor, Fasudil could decrease leukocyte recruitment and adhesion to endothelial cells, and improve the learning function after injury of cerebral ischemia/reperfusion [62]. KD025, a selective ROCK2 inhibitor, showed a dose-dependent reduction of focal cerebral ischemia-induced infarct size and improvement of ischemia outcome in mice. Interestingly, compared with nonselective ROCK inhibitors, KD025 has been demonstrated to be safer and did not induce remarkable hypotension in diabetic, aged, or female mouse [63].
A large number of ROCK inhibitors have been developed in the last few decades, which are beneficial in the outcome of ischemic stroke, spinal cord injury, and other neurological diseases, such as Alzheimer's disease and amyotrophic lateral sclerosis. It has been shown in several human autopsy and animal studies that ROCK plays a key role in the pathophysiology of spinal cord. In the mouse model of spinal cord, axon regeneration and functional recovery were significantly promoted on the treatment with nonselective ROCK inhibitor, Fasudil or Y27632 [64-66].

Taken together, ROCK has been considered to be a promising therapeutic target for stroke. There is accumulating evidence that upregulation and activation of ROCK1 and ROCK2 contribute to distinct functions on the pathogenesis of ischemic stroke and neurodegenerative diseases, which may help determine whether selective ROCK2 inhibitors could be clinically useful. Therefore, further studies are needed to develop inhibitors specifically affecting ROCK2.

\section{Acknowledgement}

The authors thank Qingyun Xiang for her helpful information on cerebral ischemia.

\section{Statement of Ethics}

Not applicable.

\section{Disclosure Statement}

The authors declare that they have no competing interests.

\section{Funding Sources}

This research was supported by Natural Science Foundation of Hefei Technology College (No. 201914KJA020). This grant was used to collect data.

\section{Author Contributions}

All authors contributed to this work. W.L., J.W., and Z.C. prepared the manuscript. All authors read and approved the final manuscript.

\section{Consent for Publication}

Not applicable.
Lu/Wen/Chen 


\section{References}

1 Lai AY, McLaurin J. Rho-associated protein kinases as therapeutic targets for both vascular and parenchymal pathologies in Alzheimer's disease. J Neurochem. 2018 Mar;144(5): 659-68.

2 Sladojevic N, Yu B, Liao JK. ROCK as a therapeutic target for ischemic stroke. Expert Rev Neurother. 2017 Dec;17(12):1167-77.

3 Tönges L, Koch JC, Bähr M, Lingor P. ROCKing Regeneration: Rho Kinase Inhibition as Molecular Target for Neurorestoration. Front Mol Neurosci. 2011 Nov; 4:39.

4 Mulherkar S, Firozi K, Huang W, Uddin MD, Grill RJ, Costa-Mattioli M, et al. RhoA-ROCK Inhibition Reverses Synaptic Remodeling and Motor and Cognitive Deficits Caused by Traumatic Brain Injury. Sci Rep. 2017 Sep; 7(1):10689.

5 Fujii M, Duris K, Altay O, Soejima Y, Sherchan P, Zhang JH. Inhibition of Rho kinase by hydroxyfasudil attenuates brain edema after subarachnoid hemorrhage in rats. Neurochem Int. 2012 Feb;60(3):327-33.

6 Wang J, Li H, Yao Y, Ren Y, Lin J, Hu J, et al. $\beta$-Elemene Enhances GAP-43 Expression and Neurite Outgrowth by Inhibiting RhoA Kinase Activation in Rats with Spinal Cord Injury. Neuroscience. 2018 Jul;383:12-21.

7 Koch JC, Tatenhorst L, Roser AE, Saal KA, Tönges L, Lingor P. ROCK inhibition in models of neurodegeneration and its potential for clinical translation. Pharmacol Ther. 2018 Sep; 189:1-21.

8 Herskowitz JH, Seyfried NT, Gearing M, Kahn RA, Peng J, Levey AI, et al. Rho kinase II phosphorylation of the lipoprotein receptor LR11/SORLA alters amyloid-beta production. J Biol Chem. 2011 Feb;286(8):6117-27.

9 Herskowitz JH, Feng Y, Mattheyses AL, Hales CM, Higginbotham LA, Duong DM, et al. Pharmacologic inhibition of ROCK2 suppresses amyloid- $\beta$ production in an Alzheimer's disease mouse model. J Neurosci. 2013 Dec;33(49):19086-98.

10 Gentry EG, Henderson BW, Arrant AE, Gearing M, Feng Y, Riddle NC, et al. Rho Kinase Inhibition as a Therapeutic for Progressive Supranuclear Palsy and Corticobasal Degeneration. J Neurosci. 2016 Jan;36(4):1316-23.

11 Henderson BW, Gentry EG, Rush T, Troncoso JC, Thambisetty M, Montine TJ, et al. Rho-associated protein kinase 1 (ROCK1) is increased in Alzheimer's disease and ROCK1 depletion reduces amyloid- $\beta$ levels in brain. J Neurochem. 2016 Aug;138(4):525-31.

12 Henderson BW, Greathouse KM, Ramdas R, Walker CK, Rao TC, Bach SV, et al. Pharmacologic inhibition of LIMK1 provides dendritic spine resilience against $\beta$-amyloid. Sci Signal. 2019 Jun;12(587):12.

13 Mahar M, Cavalli V. Intrinsic mechanisms of neuronal axon regeneration. Nat Rev Neurosci. 2018 Jun;19(6):323-37.

14 Mueller BK, Mack H, Teusch N. Rho kinase, a promising drug target for neurological dis- orders. Nat Rev Drug Discov. 2005 May;4(5): 387-98.

15 Tan HB, Zhong YS, Cheng Y, Shen X. Rho/ ROCK pathway and neural regeneration: a potential therapeutic target for central nervous system and optic nerve damage. Int J Ophthalmol. 2011;4(6):652-7.

16 Greathouse KM, Boros BD, Deslauriers JF, Henderson BW, Curtis KA, Gentry EG, et al. Distinct and complementary functions of rho kinase isoforms ROCK1 and ROCK2 in prefrontal cortex structural plasticity. Brain Struct Funct. 2018 Dec;223(9):4227-41.

17 Swanger SA, Mattheyses AL, Gentry EG, Herskowitz JH. ROCK1 and ROCK2 inhibition alters dendritic spine morphology in hippocampal neurons. Cell Logist. 2016 Jan;5(4): e1133266.

18 Dontchev VD, Letourneau PC. Growth cones integrate signaling from multiple guidance cues. J Histochem Cytochem. 2003 Apr;51(4): 435-44.

19 Ben-Zvi A, Ben-Gigi L, Yagil Z, Lerman O, Behar O. Semaphorin3A regulates axon growth independently of growth cone repulsion via modulation of TrkA signaling. Cell Signal. 2008 Mar;20(3):467-79.

20 Sahin M, Greer PL, Lin MZ, Poucher H, Eberhart J, Schmidt S, et al. Eph-dependent tyrosine phosphorylation of ephexin 1 modulates growth cone collapse. Neuron. 2005 Apr; 46(2):191-204.

21 Lin X, Ogiya M, Takahara M, Yamaguchi W, Furuyama T, Tanaka H, et al. Sema4D-plexin-B1 implicated in regulation of dendritic spine density through RhoA/ROCK pathway. Neurosci Lett. 2007 Nov;428(1):1-6.

22 Taniguchi Y, Amazaki M, Furuyama T, Yamaguchi W, Takahara M, Saino O, et al. Sema4D deficiency results in an increase in the number of oligodendrocytes in healthy and injured mouse brains. J Neurosci Res. 2009 Oct;87(13):2833-41.

$23 \mathrm{Gu}$ H, Yu SP, Gutekunst CA, Gross RE, Wei L. Inhibition of the Rho signaling pathway improves neurite outgrowth and neuronal differentiation of mouse neural stem cells. Int J Physiol Pathophysiol Pharmacol. 2013;5(1):11-20.

24 Piazzolla D, Meissl K, Kucerova L, Rubiolo C, Baccarini M. Raf-1 sets the threshold of Fas sensitivity by modulating Rok-alpha signaling. J Cell Biol. 2005 Dec;171(6):1013-22.

25 Zhang L, Valdez JM, Zhang B, Wei L, Chang J, Xin L. ROCK inhibitor Y-27632 suppresses dissociation-induced apoptosis of murine prostate stem/progenitor cells and increases their cloning efficiency. PLoS One. 2011 Mar; 6(3):e18271.

26 González-Forero D, Montero F, García-Morales V, Domínguez G, Gómez-Pérez L, García-Verdugo JM, et al. Endogenous Rho-kinase signaling maintains synaptic strength by stabilizing the size of the readily releasable pool of synaptic vesicles. J Neurosci. 2012 Jan; 32(1):68-84.
27 Koch JC, Tönges L, Barski E, Michel U, Bähr $\mathrm{M}$, Lingor P. ROCK2 is a major regulator of axonal degeneration, neuronal death and axonal regeneration in the CNS. Cell Death Dis. 2014 May;5(5):e1225.

28 Tönges L, Frank T, Tatenhorst L, Saal KA, Koch JC, Szego EM, et al. Inhibition of rho kinase enhances survival of dopaminergic neurons and attenuates axonal loss in a mouse model of Parkinson's disease. Brain. 2012 Nov;135(Pt 11):3355-70.

29 Moreno-López B, Sunico CR, González-Forero D. NO orchestrates the loss of synaptic boutons from adult "sick" motoneurons: modeling a molecular mechanism. Mol Neurobiol. $2011 \mathrm{Feb}$;3(1):41-66.

30 Srinivasan G, Kim JH, von Gersdorff $H$. The pool of fast releasing vesicles is augmented by myosin light chain kinase inhibition at the calyx of Held synapse. J Neurophysiol. 2008 Apr;99(4):1810-24.

31 Nelson JC, Stavoe AK, Colón-Ramos DA. The actin cytoskeleton in presynaptic assembly. Cell Adhes Migr. 2013 Jul-Aug;7(4):37987.

32 Newell-Litwa KA, Badoual M, Asmussen H, Patel H, Whitmore L, Horwitz AR. ROCK1 and 2 differentially regulate actomyosin organization to drive cell and synaptic polarity. J Cell Biol. 2015 Jul;210(2):225-42.

33 Komagome R, Kimura K, Saito M. Postnatal changes in Rho and Rho-related proteins in the mouse brain. Jpn J Vet Res. 2000 Feb;47(34):127-33.

34 Hashimoto R, Nakamura Y, Kosako H, Amano $\mathrm{M}$, Kaibuchi K, Inagaki M, et al. Distribution of Rho-kinase in the bovine brain. Biochem Biophys Res Commun. 1999 Sep; 263(2):575-9.

35 Shi J, Wu X, Surma M, Vemula S, Zhang L, Yang $Y$, et al. Distinct roles for ROCK1 and ROCK2 in the regulation of cell detachment. Cell Death Dis. 2013 Feb;4(2):e483.

36 Counts SE, Alldred MJ, Che S, Ginsberg SD, Mufson EJ. Synaptic gene dysregulation within hippocampal CA1 pyramidal neurons in mild cognitive impairment. Neuropharmacology. 2014 Apr;79:172-9.

37 Spruston N. Pyramidal neurons: dendritic structure and synaptic integration. Nat Rev Neurosci. 2008 Mar;9(3):206-21.

38 Huentelman MJ, Stephan DA, Talboom J, Corneveaux JJ, Reiman DM, Gerber JD, et al. Peripheral delivery of a ROCK inhibitor improves learning and working memory. Behav Neurosci. 2009 Feb;123(1):218-23.

39 Wang HG, Lu FM, Jin I, Udo H, Kandel ER, de Vente J, et al. Presynaptic and postsynaptic roles of NO, cGK, and RhoA in long-lasting potentiation and aggregation of synaptic proteins. Neuron. 2005 Feb;45(3):389-403.

40 Salminen A, Suuronen T, Kaarniranta K. ROCK, PAK, and Toll of synapses in Alzheimer's disease. Biochem Biophys Res Commun. 2008 Jul;371(4):587-90. 
41 Rust MB. ADF/cofilin: a crucial regulator of synapse physiology and behavior. Cell $\mathrm{Mol}$ Life Sci. 2015 Sep;72(18):3521-9.

42 Zebda N, Bernard O, Bailly M, Welti S, Lawrence DS, Condeelis JS. Phosphorylation of $\mathrm{ADF} /$ cofilin abolishes EGF-induced actin nucleation at the leading edge and subsequent lamellipod extension. J Cell Biol. 2000 Nov; 151(5):1119-28.

43 Islam SU, Ahmed MB, Lee SJ, Shehzad A, Sonn JK, Kwon OS, et al. PRP4 kinase induces actin rearrangement and epithelial-mesenchymal transition through modulation of the actin-binding protein cofilin. Exp Cell Res. 2018 Aug;369(1):158-65.

44 Huang L, He Z, Guo L, Wang H. Improvement of cognitive deficit and neuronal damage in rats with chronic cerebral ischemia via relative long-term inhibition of rho-kinase. Cell Mol Neurobiol. 2008 Aug;28(5):757-68.

45 Kumar M, Bansal N. Fasudil hydrochloride ameliorates memory deficits in rat model of streptozotocin-induced Alzheimer's disease: involvement of PI3-kinase, eNOS and NFKB. Behav Brain Res. 2018 Oct;351:4-16.

46 Liu Y, Yang X, Lei Q, Li Z, Hu J, Wen X, et al PEG-PEI/siROCK2 Protects Against A $\beta 42$ Induced Neurotoxicity in Primary Neuron Cells for Alzheimer Disease. Cell Mol Neurobiol. 2015 Aug;35(6):841-8.

47 Kalia LV, Lang AE. Parkinson's disease. Lancet. 2015 Aug;386(9996):896-912.

48 Greathouse KM, Henderson BW, Gentry EG, Herskowitz JH. Fasudil or genetic depletion of ROCK1 or ROCK2 induces anxiety-like behaviors. Behav Brain Res. 2019 Nov;373: 112083.

49 Yoshizaki K, Adachi K, Kataoka S, Watanabe A, Tabira T, Takahashi K, et al. Chronic cerebral hypoperfusion induced by right unilateral common carotid artery occlusion causes delayed white matter lesions and cognitive impairment in adult mice. Exp Neurol. 2008 Apr;210(2):585-91.
50 Hasan Z, Palani K, Rahman M, Zhang S, Syk I, Jeppsson B, et al. Rho-kinase signaling regulates pulmonary infiltration of neutrophils in abdominal sepsis via attenuation of CXC chemokine formation and Mac-1 expression on neutrophils. Shock. 2012 Mar;37(3):282-8.

51 Raad M, El Tal T, Gul R, Mondello S, Zhang Z, Boustany RM, et al. Neuroproteomics approach and neurosystems biology analysis: ROCK inhibitors as promising therapeutic targets in neurodegeneration and neurotrauma. Electrophoresis. 2012 Dec;33(24):365968.

52 Weiss S, Frischknecht K, Greutert H, Payeli S, Steffel J, Lüscher TF, et al. Different migration of vascular smooth muscle cells from human coronary artery bypass vessels. Role of Rho/ ROCK pathway. J Vasc Res. 2007;44(2):14956.

53 Chen ZY, Yao WJ. [Role of Rho/ROCK in the migration of vascular smooth muscle cells]. Sheng Li Ke Xue Jin Zhan. 2013 Aug;44(4): 269-74.

54 Wu J, Li J, Hu H, Liu P, Fang Y, Wu D. Rhokinase inhibitor, fasudil, prevents neuronal apoptosis via the Akt activation and PTEN inactivation in the ischemic penumbra of rat brain. Cell Mol Neurobiol. 2012 Oct;32(7): 1187-97.

55 Yan YY, Wang XM, Jiang Y, Chen H, He JT, Mang J, et al. The role of Rho/Rho-kinase pathway and the neuroprotective effects of fasudil in chronic cerebral ischemia. Neural Regen Res. 2015 Sep;10(9):1441-9.

56 Wang Y, Zheng XR, Riddick N, Bryden M, Baur W, Zhang X, et al. ROCK isoform regulation of myosin phosphatase and contractility in vascular smooth muscle cells. Circ Res. 2009 Feb;104(4):531-40.

57 Sumi T, Matsumoto K, Nakamura T. Specific activation of LIM kinase 2 via phosphorylation of threonine 505 by ROCK, a Rho-dependent protein kinase. J Biol Chem. 2001 Jan; 276(1):670-6.
58 Bernard O. Lim kinases, regulators of actin dynamics. Int J Biochem Cell Biol. 2007;39(6): 1071-6.

59 Zhang YM, Bo J, Taffet GE, Chang J, Shi J, Reddy AK, et al. Targeted deletion of ROCK1 protects the heart against pressure overload by inhibiting reactive fibrosis. FASEB J. 2006 May;20(7):916-25.

60 Hiroi Y, Noma K, Kim HH, Sladojevic $\mathrm{N}$, Tabit CE, Li Y, et al. Neuroprotection Mediated by Upregulation of Endothelial Nitric Oxide Synthase in Rho-Associated, Coiled-Coil-Containing Kinase 2 Deficient Mice. Circ J. 2018 Mar;82(4):1195204.

61 Rikitake Y, Kim HH, Huang Z, Seto M, Yano $\mathrm{K}$, Asano $\mathrm{T}$, et al. Inhibition of Rho kinase (ROCK) leads to increased cerebral blood flow and stroke protection. Stroke. 2005 Oct; 36(10):2251-7.

62 Wang QM, Stalker TJ, Gong Y, Rikitake Y, Scalia R, Liao JK. Inhibition of Rho-kinase attenuates endothelial-leukocyte interaction during ischemia-reperfusion injury. Vasc Med. 2012 Dec;17(6):379-85.

63 Lee JH, Zheng Y, von Bornstadt D, Wei Y, Balcioglu A, Daneshmand A, et al. Selective ROCK2 Inhibition In Focal Cerebral Ischemia. Ann Clin Transl Neurol. 2014 Jan;1(1) 2-14.

64 Forgione N, Fehlings MG. Rho-ROCK inhibition in the treatment of spinal cord injury. World Neurosurg. 2014 Sep-Oct;82(3-4) e535-9.

65 Boato F, Hendrix S, Huelsenbeck SC, Hofmann F, Grosse G, Djalali S, et al. C3 peptide enhances recovery from spinal cord injury by improved regenerative growth of descending fiber tracts. J Cell Sci. 2010 May;123(Pt 10) 1652-62.

66 Fournier AE, Takizawa BT, Strittmatter SM Rho kinase inhibition enhances axonal regeneration in the injured CNS. J Neurosci. 2003 Feb;23(4):1416-23. 